\title{
Astragaloside IV inhibits isoprenaline-induced cardiac fibrosis by targeting the reactive oxygen species/mitogen-activated protein kinase signaling axis
}

\author{
HONGLIANG DAI ${ }^{1}$, GUIZHI JIA ${ }^{2}$, MEILI LU ${ }^{3}$, CHUNGUANG LIANG $^{1}$, YUE WANG ${ }^{1}$ and HONGXIN WANG ${ }^{3}$ \\ ${ }^{1}$ Department of Community Health Nursing, School of Nursing; ${ }^{2}$ Department of Physiology; \\ ${ }^{3}$ Department of Pharmacology, Jinzhou Medical University, Jinzhou, Liaoning 121001, P.R. China
}

Received December 15, 2015; Accepted December 10, 2017

DOI: $10.3892 / \mathrm{mmr} .2017 .6220$

\begin{abstract}
Cardiac fibrosis is considered an important pathological mechanism in the progression of cardiac remodeling and heart failure. Astragaloside IV (AsIV) is a major active ingredient in Astragalus membranaceus. In a preliminary experiment, it was demonstrated that this naturally occurring substance exhibited cardioprotective effects via preventing cardiomyocyte hypertrophy and apoptosis. The present study aimed to investigate the effects of AsIV on $\beta$-adrenergic receptor $(\beta-\mathrm{AR})$-mediated cardiac fibrosis, and the associated mechanism. Cell Counting Kit-8 (CCK-8) assay was used to examine the proliferation of rat cardiac fibroblast (CF) cultures. Collagen I secretion was detected by ELISA. Dihydroethidium was used to determine intracellular ROS levels. Western blotting was used to examine the expression level of total and phosphorylated mitogen-activated protein kinases (MAPKs). In the present study, the effects of AsIV on $\beta$-adrenergic receptor $(\beta$-AR) -mediated cardiac fibrosis were investigated, and the associated mechanism was revealed. Isoprenaline (ISO) is a selective $\beta$-AR agonist, and treatment with AsIV significantly inhibited (ISO)-triggered cardiac fibroblast proliferation and type I collagen synthesis. In addition, ISO resulted in a significant elevation of reactive oxygen species (ROS) levels and phosphorylation of the three profibrotic MAPKs, namely extracellular signal-regulated kinase, p38MAPK and c-Jun N-terminal kinase. AsIV effectively reversed the aforementioned ISO-induced alterations. In addition, $\mathrm{N}$-acetylcysteine, a typical ROS scavenger, mimicked the inhibitory effects of AsIV on MAPK activation. The present study demonstrated that AsIV may inhibit ISO-induced cardiac fibrosis by suppressing ROS-mediated MAPK activation.
\end{abstract}

Correspondence to: Professor Hongxin Wang, Department of Pharmacology, Jinzhou Medical University, 40, Section 3, Songpo Road, Jinzhou, Liaoning 121001, P.R. China

E-mail: jyhxwang@163.com

Key words: astragaloside IV, isoprenaline, cardiac fibrosis, reactive oxygen species, mitogen-activated protein kinase

\section{Introduction}

Cardiac hypertrophy and fibrosis are two important pathological alterations that occur during the progressive development of heart failure $(1,2)$. Cardiac fibrosis refers to the excessive accumulation of extracellular matrix (ECM) and fibroblast deposition in the heart (3). Cardiac fibrosis is important in pathological cardiac remodeling and correlates with the occurrence of several fatal heart diseases, including heart failure, severe arrhythmia and sudden cardiac death $(3,4)$. It has previously been demonstrated that isoprenaline (ISO) is important in cardiac fibrosis via increasing and/or activating several effector molecules, including reactive oxygen species (ROS), p38 mitogen-activated protein kinase (p38MAPK) and extracellular signal-regulated kinase (ERK) $(3,5)$.

Astragaloside IV (AsIV) is one of the major active ingredients of the plant Astragalus membranaceus, which is referred to as HuangQi in Chinese. As an important Qi-invigorating medicinal herb, A. membranaceus is extensively used in traditional Chinese medicine for the treatment of cardiovascular diseases, hepatitis, and kidney and skin diseases $(2,6,7)$. Chemically, AsIV is a cycloartane triterpene saponin with a well-defined molecular formula, the chemical structure of which is presented in Fig. 1. It has previously been demonstrated that AsIV exhibits cardioprotective effects via its antioxidative and anti-inflammatory properties $(8,9)$. In addition, AsIV has been reported to improve cardiac function via the inhibition of cardiomyocyte hypertrophy and apoptosis $(6,8)$. However, the function of AsIV in cardiac fibrosis remains to be fully elucidated, with only one previous study, to the best of our knowledge, currently available reporting its anti-fibrotic effect in coxsackievirus-induced cardiomyopathy (10). Therefore, the present study aimed to examine whether AsIV possesses antifibrotic activity under ISO stimulation and aimed to elucidate the associated underlying molecular mechanism.

\section{Materials and methods}

Animals. A total of 80 Sprague Dawley rat pups (age, 1-3 days; weight, $7 \pm 2 \mathrm{~g}, 40$ male, 40 female), provided by the Animal Center of Jinzhou Medical University, were used for the current study. Rat pups, together with their mothers, were maintained 
at standard conditions with temperature at $25 \pm 1^{\circ} \mathrm{C}$, and relative humidity at $70 \pm 10 \%$. Animals were given free access to food and water and maintained under a 12/12 h light/dark cycle. The experiments were performed in accordance with the U.S. National Institute of Health guidelines for the Use of Laboratory Animals and ethical approval was granted by the Jinzhou Medical University Committee on Animal Care.

Cardiac fibroblast (CF) culture. The rats were anesthetized by ether inhalation and euthanized by cervical dislocation. Primary cultures of CFs were prepared as previously described, following the method for culture of cardiomyocytes (2), with the exception that pre-attached CFs rather than cardiomyocytes were collected for use. CFs were identified by their specific marker vimentin. Dispersed CFs were cultured in Dulbecco's modified Eagle's medium (DMEM; Corning Incorporated, Corning, NY, USA) supplemented with $10 \%$ fetal bovine serum (GE Healthcare Life Sciences, Logan, UT, USA) in a humidified atmosphere of $5 \% \mathrm{CO}_{2}$, at $37^{\circ} \mathrm{C}$.

CF proliferation assay. CFs were digested with $0.25 \%$ trypsin and ethylene diamine tetraacetic acid, then cultured in 96-well plates in the presence and absence of $100 \mu \mathrm{M}$ AsIV (>98\% purity; Nanjing Jingzhu Bio-Technology Co., Ltd., Nanjing, China) or the specific inhibitor of JNK, SP600125 (10 $\mu \mathrm{M}$, Sigma-Aldrich, Merck KGaA, Darmstadt, Germany) for $30 \mathrm{~min}$ prior to treatment with $10 \mu \mathrm{M}$ ISO (Sigma-Aldrich; Merck KGaA). Following incubation for $24 \mathrm{~h}$ in a humidified atmosphere of $5 \% \mathrm{CO}_{2}$, at $37^{\circ} \mathrm{C}$, the supernatant was removed, and $100 \mu \mathrm{l}$ DMEM containing $10 \mu \mathrm{l}$ Cell Counting Kit-8 (Dojindo Molecular Technologies, Inc., Kumamoto, Japan) was added to each well for a further $4 \mathrm{~h}$ at $37^{\circ} \mathrm{C}$. The optical density was measured at a wavelength of $450 \mathrm{~nm}$ using an automated ELISA plate reader (Thermo Fisher Scientific, Inc., Waltham, MA, USA).

ELISA. The secretion of collagen type I by CFs was measured using a commercial ELISA kit (catalog no. CSB-E 12732r; Cusabio Biotech Co., Ltd., Wuhan, China) according to the manufacturer's protocol.

Measurement of intracellular ROS. Dihydroethidium (DHE; Molecular Probes; Thermo Fisher Scientific, Inc.) was used to determine intracellular ROS levels in cultured CFs. Briefly, CFs were incubated with $10 \mu \mathrm{M} \mathrm{DHE}$ for $30 \mathrm{~min}$ at $37^{\circ} \mathrm{C}$ in the dark. Cells were then washed 3 times with phosphate-buffered saline (PBS) to remove unincorporated dye. CFs loaded with DHE were then treated with $100 \mu \mathrm{M}$ AsIV for $30 \mathrm{~min}$, followed by treatment with $10 \mu \mathrm{M}$ ISO for an additional $30 \mathrm{~min}$. ROS levels were subsequently analyzed using an inverted fluorescence microscope (Leica Microsystems GmbH, Wetzlar, Germany).

Western blotting. Primary cultures of cardiac fibroblasts were pretreated with $100 \mu \mathrm{M}$ AsIV or $10 \mathrm{mM}$ N-acetylcysteine (NAC, Sigma-Aldrich; Merck KGaA) for 30 min, then treated with $10 \mu \mathrm{M}$ ISO for a further $24 \mathrm{~h}$ in a humidified atmosphere of $5 \% \mathrm{CO}_{2}$ at $37^{\circ} \mathrm{C}$. Cells were washed with cold PBS and harvested with freshly prepared lysis buffer [150 mM NaCl, $50 \mathrm{mM}$ Tris- $\mathrm{HCl}, \mathrm{pH} 8.0,0.1 \%$ sodium dodecyl sulfate, $1 \%$ Triton X-100 and $1 \%$ proteinase inhibitors (Sigma-Aldrich, Merck KGaA)]. Following quantification of total protein by Lowry's method, equal amounts of protein $(50 \mu \mathrm{g})$ were subjected to $10 \%$ sodium dodecyl sulfate-polyacrylamide gel electrolysis and blotted onto a polyvinylidene fluoride membrane. The membrane was blocked with $1 \%$ bovine serum albumin (Sigma-Aldrich; Merck $\mathrm{KGaA}$ ) for $1 \mathrm{~h}$ and then incubated overnight at $4^{\circ} \mathrm{C}$ with the primary rabbit anti-rat polyclonal antibodies against ERK (1:1,000; catalog no. sc-292838; Santa Cruz Biotechnology, Inc., Dallas, TX, USA), phosphorylated (p)-ERK (1:1,000; catalog no. sc-101760; Santa Cruz Biotechnology, Inc.), p38MAPK, (1:800; catalog no. 21683; Signalway Antibody LLC, College Park, ML, USA), p-p38MAPK (1:800; catalog no. 11581; Signalway Antibody LLC), c-Jun N-terminal kinase (JNK; 1:800; catalog no. 21241; Signalway Antibody LLC) and p-JNK, (1:800; catalog no. sc-135642; Santa Cruz Biotechnology, Inc.). Following $2 \mathrm{~h}$ incubation at room temperature with the polyclonal horseradish peroxidase-conjugated secondary goat anti-rabbit antibody (1:2,500; catalog no. sc-2004; Santa Cruz Biotechnology, Inc.), the bands were visualized by an enhanced chemiluminescence reagent (Thermo Fisher Scientific, Inc.). Optical density for each band was assessed using Quantity One software, version 4.6.9 (Bio-Rad Laboratories, Inc., Hercules, CA, USA).

Statistical analysis. Data are presented as the mean \pm standard error of the mean, from at least three independent experiments. One-way analysis of variance followed by Fisher's least significant difference test was used to compare groups by use of SPSS version 17.0 software (SPSS, Inc., Chicago, IL, USA). $\mathrm{P}<0.05$ was considered to indicate a statistically significant difference.

\section{Results}

AsIV attenuates ISO-induced CF proliferation and type I collagen synthesis. To evaluate the effects of AsIV on ISO-induced cardiac fibrosis, CF proliferation and type I collagen levels were examined. As presented in Fig. 2A, $\mathrm{CF}$ proliferation was significantly increased upon ISO stimulation for $24 \mathrm{~h}$. Treatment with AsIV $(100 \mu \mathrm{M})$ inhibited ISO-triggered CF proliferation. Increased collagen synthesis in ISO-treated cultured CFs was also significantly decreased with application of AsIV (Fig. 2B).

JNK mediates ISO-induced CF proliferation and type I collagen synthesis. The MAPK signaling pathway is composed of ERK, p38MAPK and JNK. Previous studies have demonstrated that ERK and $\mathrm{p} 38 \mathrm{MAPK}$ are required for $\beta$-adrenergic activation of CF proliferation $(3,5,11)$. However, it remains to be elucidated whether JNK is involved in this process. To investigate this, CF proliferation and type I collagen synthesis were measured following $24 \mathrm{~h}$ treatment with ISO in the absence or presence of the JNK inhibitor, SP600125. As presented in Fig. 3A and B, ISO-induced CF proliferation and collagen synthesis were significantly decreased by pretreatment with SP600125, indicative of a key role of JNK in ISO-induced cardiac fibrosis.

AsIV decreases ISO-induced ROS generation in CFs. AsIV is a potent antioxidant $(12,13)$. Furthermore, ROS is important 


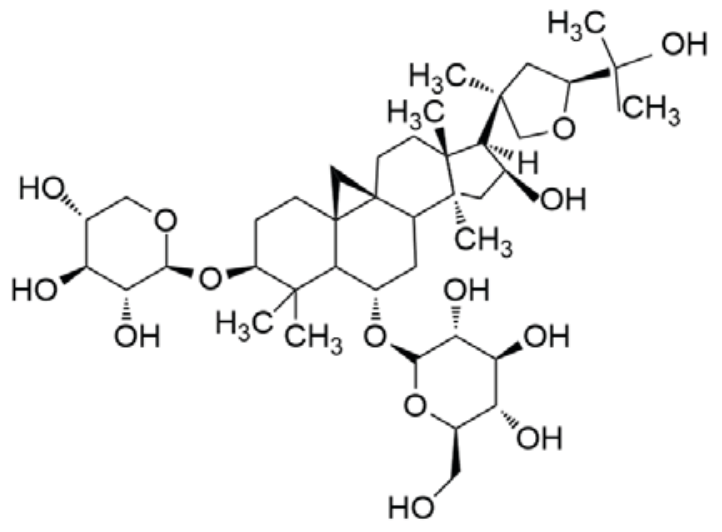

Figure 1. Chemical structure of astragaloside IV.
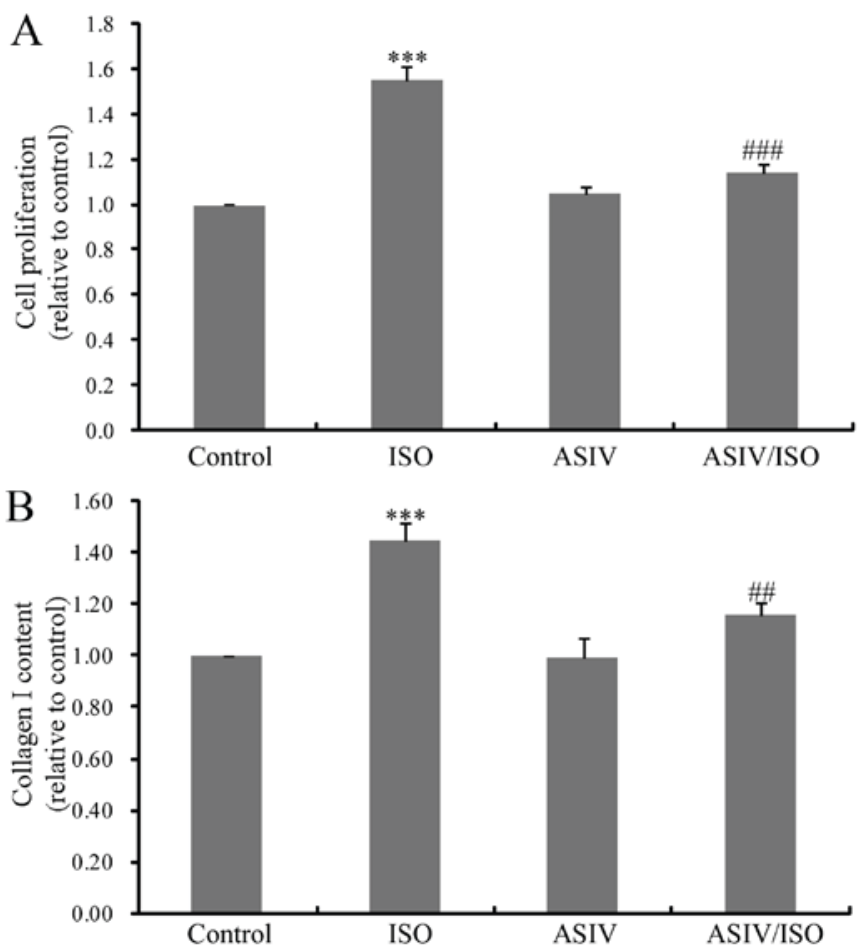

Figure 2. Effects of AsIV on ISO-induced CF proliferation and collagen synthesis. A total of $100 \mu \mathrm{M}$ AsIV was added to $10 \mu \mathrm{M}$ ISO-induced CFs to measure (A) cell proliferation and (B) type I collagen synthesis. Data are expressed as the mean \pm standard error of the mean from five independent experiments. ${ }^{* * *} \mathrm{P}<0.001$ vs. control group; ${ }^{\# \#} \mathrm{P}<0.01,{ }^{\# \# \#} \mathrm{P}<0.001$ vs. ISO group. ISO, isoprenaline; AsIV, astragaloside IV; CF, cardiac fibroblasts.

in ISO-induced cardiac fibrosis $(3,14)$. The present study therefore examined the effects of AsIV on ROS production in CFs. As presented in Fig. 4, ISO-induced ROS production was effectively decreased by pretreatment with $100 \mu \mathrm{M}$ AsIV.

AsIV and NAC attenuate ISO-induced phosphorylation of MAPK family members. In view of the important role of MAPK molecules in ISO-induced cardiac fibrosis, the present study investigated the effects of AsIV on MAPK activation. As presented in Fig. 5, ISO activated the three MAPKs, ERK, p38MAPK and JNK. Conversely, AsIV inhibited the activation of these three MAPKs. In addition, NAC, which is a typical
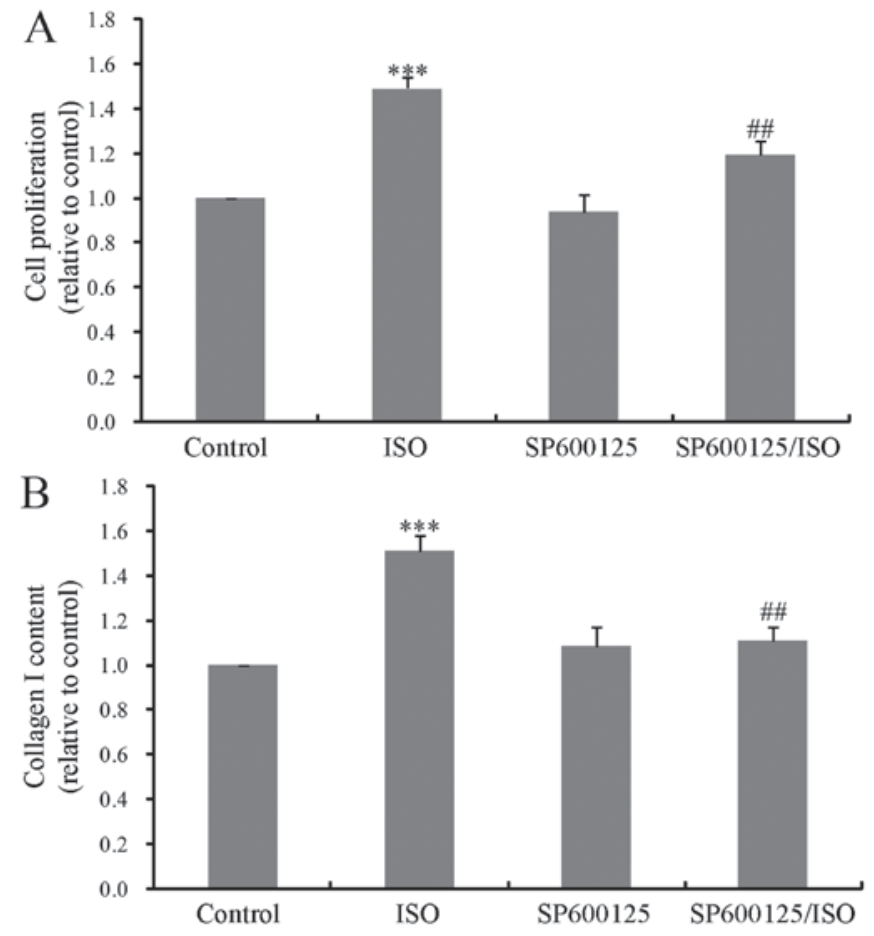

Figure 3. Effects of SP600125 on ISO-induced CF proliferation and collagen synthesis. A total of $10 \mu \mathrm{M}$ SP600125 was added to $10 \mu \mathrm{M}$ ISO-induced CFs to measure (A) cell proliferation and (B) type I collagen synthesis. Data are expressed as the mean \pm standard error of the mean from five independent experiments. ${ }^{* * *} \mathrm{P}<0.001$ vs. control group; ${ }^{\#} \mathrm{P}<0.01$ vs. ISO group. ISO, isoprenaline; CFs, cardiac fibroblasts.

ROS scavenger, exhibited similar inhibitory effects to those demonstrated by AsIV on MAPK activation.

\section{Discussion}

The present study demonstrated that AsIV may protect against ISO-induced CF proliferation and type I collagen synthesis. The underlying mechanism involved inhibition of ROS production and the resultant downregulation of MAPK signaling. To the best of our knowledge, this study is the first to reveal the antifibrotic effect, and associated mechanism, of AsIV in response to ISO stimulation.

Cardiac fibrosis is characterized by enhanced CF proliferation and excessive production and deposition of the ECM, of which $\sim 85 \%$ is composed of type I collagen $(15,16)$. This consequently results in myocardial stiffness, impaired diastolic function and cardiac failure $(17,18)$. Antifibrotic mechanisms are therefore regarded as effective therapeutic strategies for the treatment of various cardiovascular diseases. It has previously been demonstrated that AsIV exerts therapeutic effects on fibrosis in several disorders, including chronic kidney disease (19), systemic sclerosis (20), liver fibrosis (21), and coxsackievirus B3-induced cardiomyopathy (10). However, its effect on $\beta$-adrenergic receptor-induced cardiac fibrosis remains to be fully elucidated. The present study therefore investigated the effects of AsIV on ISO-mediated cardiac fibrosis and the associated signaling transduction. The results demonstrated that pretreatment with AsIV significantly inhibited ISO-induced CF proliferation and type I collagen synthesis, thus suggesting that AsIV is a promising agent 


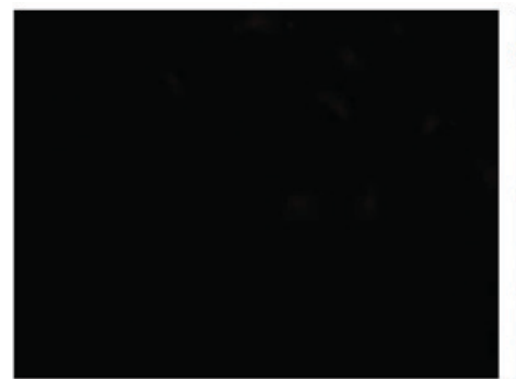

Control

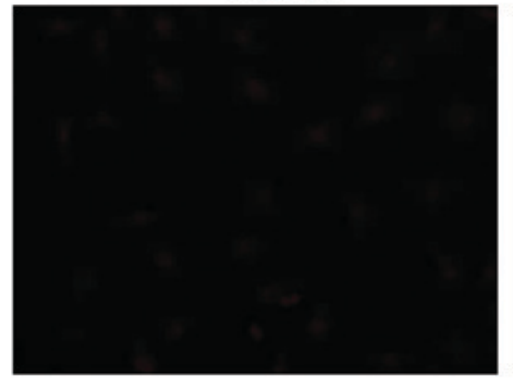

ASIV

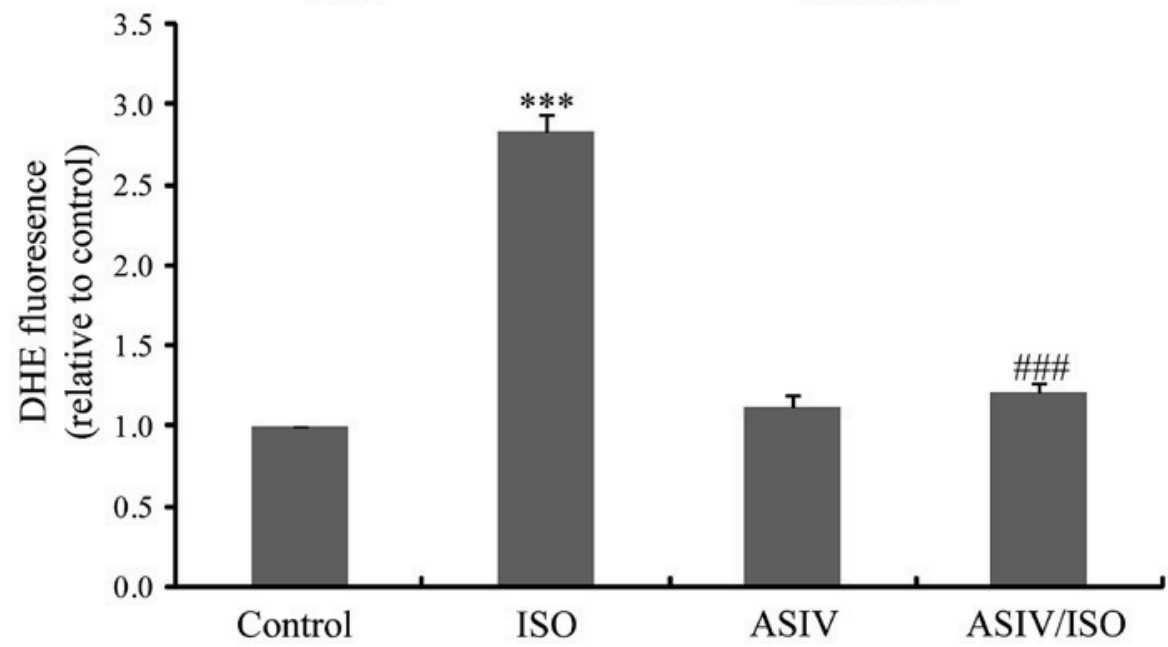

Figure 4. Effects of AsIV on ISO-induced reactive ROS levels in CFs. A total of $100 \mu \mathrm{M}$ AsIV was added to $10 \mu \mathrm{M}$ ISO-induced CFs to measure ROS levels, as detected by DHE fluorescence. Data are expressed as the mean \pm standard error of the mean from five independent experiments. ${ }^{* * *} \mathrm{P}<0.001 \mathrm{vs.}$ control group; ${ }^{\# \#} \mathrm{P}<0.001$, vs. ISO group. Magnification, $\mathrm{x} 200$. ISO, isoprenaline; ROS, reactive oxygen species; AsIV, astragaloside IV; CFs, cardiac fibroblasts; DHE, dihydroethidium.

for the prevention and treatment of cardiac fibrosis under increased sympathetic drive.

Oxidative stress is important in the development and progression of diverse cardiac disorders, including cardiac fibrosis (14), hypertrophy (22), apoptosis (23), inflammation (24) and resultant heart failure $(25,26)$. It has previously been demonstrated that ROS may modulate ECM remodeling by mediating $\mathrm{CF}$ function and stimulating collagen turnover (26). Antioxidative mechanisms may prevent numerous adverse cardiovascular events, including cardiac fibrosis. GKT137831 (14), oleanolic acid (27), 3,3'-diindolymethane (28) and magnolol (29), have been demonstrated to attenuate cardiac fibrosis via antioxidative bioactivity. The antioxidative capacity of AsIV has increasingly been identified by previous studies $(12,13)$. Data from the present study revealed that application of AsIV effectively suppressed ROS accumulation. It was therefore concluded that the antifibrotic effects of AsIV may depend on its antioxidative activity.
One important consequence of oxidative stress is the phosphorylation of MAPK proteins, including ERK, p38MAPK and JNK. MAPK activation has been observed in cardiac fibrosis in response to oxidative stress triggered by angiotensin II (30-33). In addition, it has been suggested that ISO induces cardiac fibrosis via activation of p38MAPK and ERK $(3,5)$. To elucidate the involvement of JNK in ISO-induced cardiac fibrosis, the effect of SP600125, a selective inhibitor of JNK, was investigated. The results indicated that SP600125 inhibited CF proliferation and type I collagen synthesis, suggesting a similarly indispensible role of JNK in ISO-triggered cardiac fibrosis, as that observed of ERK and p38MAPK. Furthermore, data from the present study revealed that the phosphorylation of the three pro-fibrotic MAPK molecules was abrogated by application of AsIV. In addition, the inhibitory effects of AsIV on MAPK signaling were mimicked by the selective ROS inhibitor, NAC; thus, indicating that AsIV inhibits MAPK signaling in a ROS-dependent manner. 

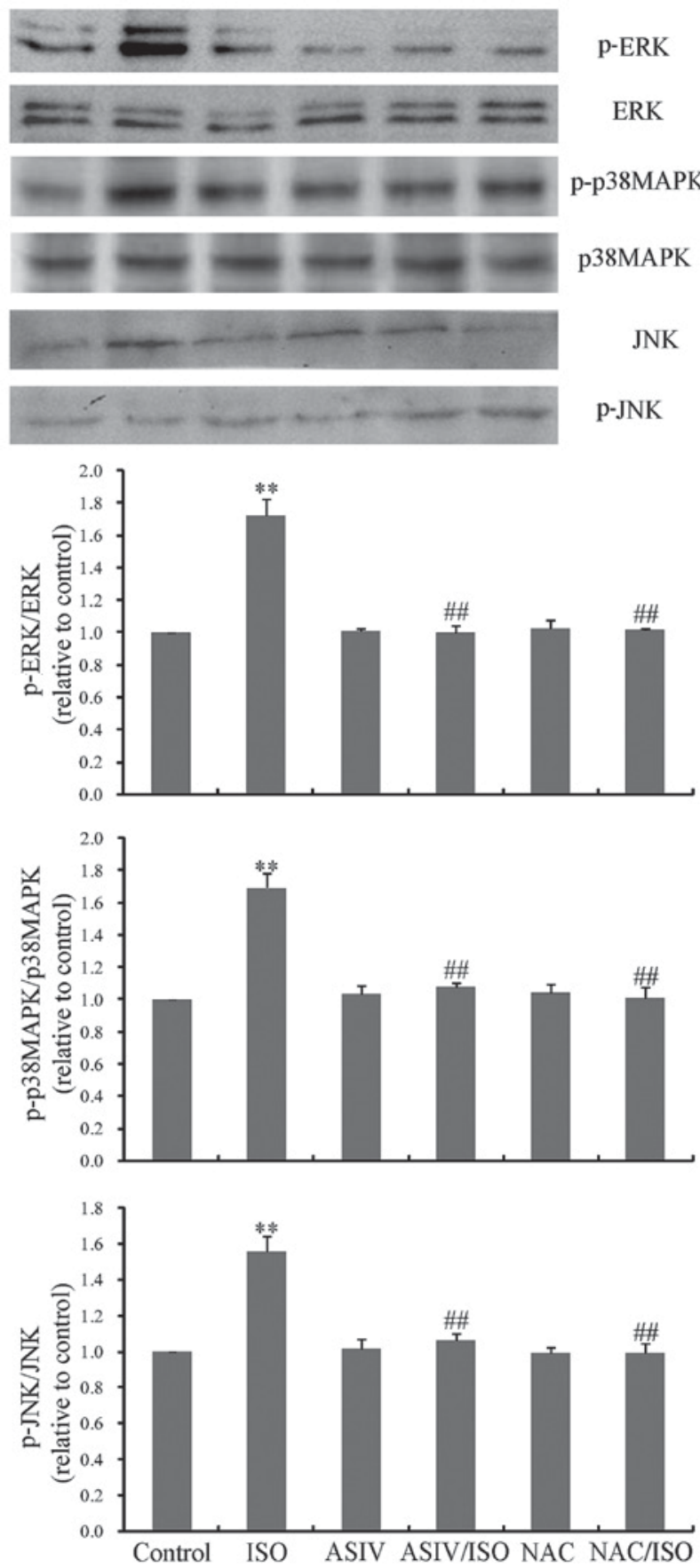

Figure 5. Effects of AsIV and NAC on ISO-induced activation of the MAPK family. Activation was measured by analyzing the expression levels of phosphorylated proteins, as detected by western blotting. A total of $100 \mu \mathrm{M}$ AsIV was added to $10 \mu \mathrm{M}$ ISO-induced CFs to measure activation of ERK, p38MAPK and JNK MAPK signaling pathways. This was repeated with the addition of $10 \mathrm{mM}$ NAC to $10 \mu \mathrm{M}$ ISO-induced CFs. Data are expressed as the mean \pm standard error of the mean from three independent experiments. ${ }^{* *} \mathrm{P}<0.01$ vs. control group; ${ }^{\# \#} \mathrm{P}<0.01$ vs. ISO group. ERK, extracellular signal-regulated kinase; p38MAPK, p38 mitogen-activated protein kinase; JNK, c-Jun N-terminal kinase; p, phosphorylated; AsIV, astragaloside IV; $\mathrm{CFs}$, cardiac fibroblasts; NAC, N-acetylcysteine; ISO, isoprenaline.

In conclusion, the present study demonstrated that AsIV effectively inhibited ISO-induced CF proliferation and type I collagen synthesis via attenuation of ROS-mediated MAPK signaling activation. These findings may aid to further elucidate the protective role and underlying molecular mechanism of AsIV in the cardiovascular system.

\section{Acknowledgements}

The present study was supported by the National Natural Science Foundation of China (grant no. 81374008), the Natural Science Foundation of Liaoning Province (grant no. 2015020360) and the President Foundation, Aohongboze Foundation of Liaoning Medical University (grant no. XZJJ20140111). The authors would like to thank Dr Guannan Wang (Jinzhou Medical University, Jinzhou, China) for his assistance in drawing the chemical structure of AsIV.

\section{References}

1. Yu LM and Xu Y: Epigenetic regulation in cardiac fibrosis. World J Cardiol 7: 784-791, 2015.

2. Dai H, Jia G, Liu X, Liu Z and Wang H: Astragalus polysaccharide inhibits isoprenaline-induced cardiac hypertrophy via suppressing $\mathrm{Ca}^{2+}$-mediated calcineurin/NFATc3 and CaMKII signaling cascades. Environ Toxicol Pharmacol 38: 263-271, 2014.

3. Lu H, Tian A, Wu J, Yang C, Xing R, Jia P, Yang L, Zhang Y, Zheng $X$ and Li Z: Danshensu inhibits $\beta$-adrenergic receptors-mediated cardiac fibrosis by ROS/p38 MAPK axis. Biol Pharm Bull 37: 961-967, 2014.

4. Morita N, Mandel WJ, Kobayashi Y and Karagueuzian HS: Cardiac fibrosis as a determinant of ventricular tachyarrhythmias. J Arrhythm 30: 389-394, 2014.

5. Kim J, Eckhart AD, Eguchi S and Koch WJ: Beta-adrenergic receptor-mediated DNA synthesis in cardiac fibroblasts is dependent on transactivation of the epidermal growth factor receptor and subsequent activation of extracellular signal-regulated kinases. J Biol Chem 277: 32116-32123, 2002.

6. Yang J, Wang HX, Zhang YJ, Yang YH, Lu ML, Zhang J, Li ST, Zhang SP and Li G: Astragaloside IV attenuates inflammatory cytokines by inhibiting TLR4/NF-KB signaling pathway in isoproterenol-induced myocardial hypertrophy. J Ethnopharmacol 150: 1062-1070, 2013.

7. Jiang X, Cao X, Huang Y, Chen J, Yao X, Zhao M, Liu Y, Meng J, Li P, Li Z, et al: Effects of treatment with Astragalus Membranaceus on function of rat leydig cells. BMC Complement Altern Med 15: 261, 2015.

8. Mei M, Tang F, Lu M, He X, Wang H, Hou X, Hu J, Xu C and Han R: Astragaloside IV attenuates apoptosis of hypertrophic cardiomyocyte through inhibiting oxidative stress and calpain-1 activation. Environ Toxicol Pharmacol 40: 764-773, 2015.

9. Zhao P, Wang Y, Zeng S, Lu J, Jiang TM and Li YM: Protective effect of astragaloside IV on lipopolysaccharide-induced cardiac dysfunction via downregulation of inflammatory signaling in mice. Immunopharmacol Immunotoxicol 37: 428-433, 2015.

10. Chen P, Xie Y, Shen E, Li GG, Yu Y, Zhang CB, Yang Y, Zou Y, Ge J, Chen R and Chen H: Astragaloside IV attenuates myocardial fibrosis by inhibiting TGF- $\beta 1$ signaling in coxsackievirus B3-induced cardiomyopathy. Eur J Pharmacol 658: 168-174, 2011.

11. Liu N, Xing R, Yang C, Tian A, Lv Z, Sun N, Gao X, Zhang Y and Li Z: HIP-55/DBNL-dependent regulation of adrenergic receptor mediates the ERK1/2 proliferative pathway. Mol Biosyst 10: 1932-1939, 2014.

12. Lu Y, Li S, Wu H, Bian Z, Xu J, Gu C, Chen X and Yang D: Beneficial effects of astragaloside IV against angiotensin II-induced mitochondrial dysfunction in rat vascular smooth muscle cells. Int J Mol Med 36: 1223-1232, 2015.

13. Wang SG, Xu Y, Xie H, Wang W and Chen XH: Astragaloside IV prevents lipopolysaccharide-induced injury in H9C2 cardiomyocytes. Chin J Nat Med 13: 127-132, 2015.

14. Somanna NK, Valente AJ, Krenz M, Fay WP, Delafontaine P and Chandrasekar B: The Nox1/4 dual inhibitor GKT137831 or Nox4 knockdown Inhibits Angiotensin-II-Induced adult mouse cardiac fibroblast proliferation and migration. AT1 physically associates with Nox4. J Cell Physiol 231: 1130-1141, 2016.

15. Porter KE and Turner NA: Cardiac fibroblasts: At the heart of myocardial remodeling. Pharmacol Ther 123: 255-278, 2009.

16. Ye Y, Lv X, Wang MH, Zhu J, Chen SQ, Jiang CY and Fu GS: Alendronate prevents angiotensin II-induced collagen I production through geranylgeranylation-dependent RhoA/Rho kinase activation in cardiac fibroblasts. J Pharmacol Sci 129: 205-209, 2015. 
17. Segura AM, Frazier OH and Buja LM: Fibrosis and heart failure. Heart Fail Rev 19: 173-185, 2014.

18. Tao H, Yang JJ, Shi KH, Deng ZY and Li J: DNA methylation in cardiac fibrosis: New advances and perspectives. Toxicology 323: 125-129, 2014.

19. Che X, Wang Q, Xie Y, Xu W, Shao X, Mou S and Ni Z: Astragaloside IV suppresses transforming growth factor- $\beta 1$ induced fibrosis of cultured mouse renal fibroblasts via inhibition of the MAPK and NF- $\mathrm{BB}$ signaling pathways. Biochem Biophys Res Commun 464: 1260-1266, 2015.

20. Qi Q, Mao Y, Yi J, Li D, Zhu K and Cha X: Anti-fibrotic effects of Astragaloside IV in systemic sclerosis. Cell Physiol Biochem 34: 2105-2116, 2014

21. Li X, Wang X, Han C, Wang X, Xing G, Zhou L, Li G and Niu Y: Astragaloside IV suppresses collagen production of activated hepatic stellate cells via oxidative stress-mediated p38 MAPK pathway. Free Radic Biol Med 60: 168-176, 2013.

22. Zhang C, Wang F, Zhang Y, Kang Y, Wang H, Si M, Su L, Xin X, Xue F, Hao F, et al: Celecoxib prevents pressure overload-induced cardiac hypertrophy and dysfunction by inhibiting inflammation, apoptosis and oxidative stress. J Cell Mol Med 20: 116-127, 2016.

23. Liu B, Zhang J, Liu W, Liu N, Fu X, Kwan H, Liu S, Liu B, Zhang S, Yu Z and Liu S: Calycosin inhibits oxidative stress-induced cardiomyocyte apoptosis via activating estrogen receptor- $\alpha / \beta$. Bioorg Med Chem Lett 26: 181-185, 2016.

24. Kayama Y, Raaz U, Jagger A, Adam M, Schellinger IN, Sakamoto M, Suzuki H, Toyama K, Spin JM and Tsao PS Diabetic cardiovascular disease induced by oxidative stress. Int J Mol Sci 16: 25234-25263, 2015.

25. Fournier P, Fourcade J, Roncalli J, Salvayre R, Galinier M and Causse E: Homocysteine in chronic heart failure. Clin Lab 61: 1137-1145, 2015.
26. Murdoch CE, Zhang M, Cave AC and Shah AM: NADPH oxidase-dependent redox signalling in cardiac hypertrophy, remodelling and failure. Cardiovasc Res 71: 208-215, 2006.

27. Liao HH, Zhang N, Feng H, Zhang N, Ma ZG, Yang Z, Yuan Y, Bian ZY and Tang QZ: Oleanolic acid alleviated pressure overload-induced cardiac remodeling. Mol Cell Biochem 409: 145-154, 2015.

28. Yao Z, Hu W, Yin S, Huang Z, Zhu Q, Chen J, Zang Y, Dong L and Zhang J: 3,3'-Diindolymethane ameliorates adriamycin-induced cardiac fibrosis via activation of a BRCA1-dependent anti-oxidant pathway. Pharmacol Res 70: 139-146, 2013.

29. Liou JY, Chen YL, Loh SH, Chen PY, Hong CY, Chen JJ, Cheng TH and Liu JC: Magnolol depresses urotensin-II-induced cell proliferation in rat cardiac fibroblasts. Clin Exp Pharmacol Physiol 36: 711-716, 2009.

30. Chan P, Liu JC, Lin LJ, Chen PY, Cheng TH, Lin JG and Hong HJ: Tanshinone IIA inhibits angiotensin II-induced cell proliferation in rat cardiac fibroblasts. Am J Chin Med 39: 381-394, 2011.

31. Zhang W, Chen XF, Huang YJ, Chen QQ, Bao YJ and Zhu W: $2,3,4^{\prime}, 5$-Tetrahydroxystilbene-2-O- $\beta$-D-glucoside inhibits angiotensin II-induced cardiac fibroblast proliferation via suppression of the reactive oxygen species-extracellular signal-regulated kinase 1/2 pathway. Clin Exp Pharmacol Physiol 39: 429-437, 2012.

32. Yu M, Zheng Y, Sun HX and Yu DJ: Inhibitory effects of enalaprilat on rat cardiac fibroblast proliferation via ROS/P38MAPK/TGF- $\beta 1$ signaling pathway. Molecules 17: 2738-2751, 2012

33. Shyu KG, Wang BW, Chen WJ, Kuan P and Lin CM: Angiotensin II mediates urotensin II expression by hypoxia in cultured cardiac fibroblast. Eur J Clin Invest 42: 17-26, 2012. 